\title{
NEUE LITERATUR
}

Bu,TNKs, L.R . 1955. Photosynthesis and productivity of littoral marine algae. Journ. Marine Res. 14, 363-373.

VinCENT, D. L. 1956. The preparation of sodium alginate from rockweed. Canadian Journ. Res. 34, $220-226$.

Bendz, G. 1956. Galic acid isolated from water extracts of litter from Acer pseudoplatanus. Physiologia plantar. 9, 243-246.

Pascat, M. P. 1955. Aportaciones al conocimiento de variedades interesantes de algodón para los regadíos Aragoneses.

Boletín Nr. 33 del Inst. Nac. Investig. Agron. 376-422.

Simpson, D. M. and E. N. DunCan 1956. Cotton pollen dispersal by insects. Agron. Journ. 48, 305-308.

SCHANDER, H. 1956 Über die Ursachen von Gewichtsunterschieden bei Samen von Kernobst (Apfel und Birne). II. Der Einfluß verschiedener Erb- und Umweltfaktoren auf die Beziehungen zwischen Samen und Frucht. (Beiträge zur Physiologie der Fruchtentwicklung). Ztschr. f. Pfanzenzüchtg. 36, $31-80$.

GaLUn, EsRA 1956. Effect of seed treatment on sex expression in the cucumber. Experientia (Basel) 12, 218-219.

Misra, Gadadhar 1955. Photoperiodism in rice. VIII. Effects of short photoperiod on four varieties of early-winter rice.

Proceed. Nat. Inst. Sci. India, Part B, 21, 201-209.

Skok, JoHN AND N. J. SCULIY 1955. Nature of the photoperiodic responses of buckwheat. Bot. Gaz. 117, 134-141.

Downs, R. J. AND H. A. BoRTHWick 1956. Effects of photoperiod on growth of trees. Bot. Gaz. 11\%, 310-326.

Lefvadoux, L. 1956. Les populations sauvages et cultivées de Vitis vinifera L.: Annales Inst. Nat. Rech. agronom., Sér. B, 5, 59-118.

Mayr, ERNst 1956. Geographical character gradients and climatic adaption. Evolution (Lancaster, Pa.) 10, 105-108.

Hanke, E. UND H. KaISER 1956. Untersuchungen über den Einfluß eines künstlichen Windschutzstreifens auf den Ertrag von Zuckerrüben im Jahre 1954. Ztschr. f. Acker- und Pfanzenbau. 102, 81-100.

JoHnson, S. P. AND WAYNE C. HALl 1955. Further studies on vegetative and fruiting responses of tomatoes to high temperature and light intensity. Bot. Gaz. 11\%, 100-113.

STEINECK, O. 1956. Tageslänge und Knollenbildung bei Kultursorten der Kartoffel. Ztschr. f. Pfanzenzüchtung. 26, 197-213.

Maded, P. 1956. La nature et les causes du boulage chez la pomme de terre. Annales de l'Amélioration des Plantes. 6, 151-169.

Madec, P. et P. Perennec 1954. Contribution à l'étude de la tubérisation chez la pomme de terre. Annales de l'Amélioration des Plantes. 4, 449-467.

Madec, P. et P. Perenneo 1955. Les possibilités d'évolution des germes de la pomme de terre et leurs conséquences. Annales de l'Amélioration des Plantes. 5. 555-574. 